Check for updates

1 Durham University

2 University of Southampton

3 University Hospital Southampton NHS Foundation Trust

Cite this as: BMJ 2021;375:n2933 http://dx.doi.org/10.1136/bmi.n2933

Published: 26 November 2021

\section{Navigating the social identity of long covid}

\author{
Julie Van de Vyver, ${ }^{1}$ Ana C. Leite, ${ }^{1}$ Nisreen A. Alwan², 3
}

Long covid is an urgent global public health problem. In the UK alone, experiencing it for a year or more is estimated to affect 405000 people, with higher prevalence in deprived groups. ${ }^{1}$ Medical research into the pathophysiology of long covid, and how it can be treated is urgently needed, but it is also essential to consider social factors that may be implicated in the recovery of the millions of people with long covid across the world. It is not possible to holistically understand any individual's experiences, without understanding their connection and place within the broader community around them. Research shows that social connections and belonging are essential determinants of health and wellbeing. ${ }^{2}$

\section{Stigma, isolation, and identity loss in long covid}

The particular features of long covid as a long lasting condition with multifactorial presentation, often without firm medical diagnoses, put patients at substantial risk of experiencing stigma. ${ }^{3}$ The idea that "invisible" chronic illnesses with poorly characterised underlying processes may lead to stigma is not new. Stigma not only affects people's quality of life, but can lead to avoidance of medical help-seeking, which may exacerbate illness. People with long covid may experience "double stigma" if they belong to certain groups in society, for example ethnic minorities, women, immigrants and refugees, economically disadvantaged groups, and those with learning disabilities, through implicit biases in health, social care, and employment settings. ${ }^{45}$

Due to the nature of the condition, people living with long covid are additionally at risk of experiencing isolation. The most prevalent symptom of long covid, debilitating fatigue, reduces patients' access to social contact and connection with their communities, and thereby their access to social support. Also, people living with long covid may experience some degree of identity loss. Many people with long covid were previously active, and the illness has unavoidably affected their ability to work, exercise, care for their families, and relate to others. This likely has a huge impact on their professional identity, abled identity, and identity as an active member of society, whether a child or adult. It is crucial to understand how stigma, isolation, and social identity shape people's experiences of long covid, and their recovery or disease progression journeys.

\section{Social identity approach to health}

Social identity theory highlights that we hold multiple identities derived from our group memberships and that these shape our attitudes, beliefs, and behaviours. Groups play a crucial role in helping us to make sense of the world, to navigate threats, and to capitalise on opportunities. A growing body of research demonstrates reliable impacts of group memberships and social identities on individual wellbeing and health - where groups can provide a "social cure" against illness, by enhancing a sense of belongingness, control and agency,

self-affirmation, meaning and purpose, and collective self-efficacy and support. For example, research has demonstrated that greater social connectedness decreases symptoms of depression, stress, anxiety, and loneliness. ${ }^{6-11}$ Taking a social identity approach can help us design holistic support mechanisms for people living with long covid.

\section{The complexity of social identity processes in the} context of long covid

The social identity processes involved in long covid are complex, and urgently require rigorous research and attention. For example, it is difficult to derive a positive meaning from one's social identity, if one's social group is subjected to stigma, or indeed if they are unable to connect to their previously positive identities due to illness-related identity loss. Furthermore, the benefits of joining different support groups may vary, depending on whether they promote collective efficacy vs. helplessness.

Additionally, given existing structural inequalities, deprived and minority communities may have a narrower range of social support at their disposal. It is likely that the positive impact of social group memberships may operate less effectively among those who need it the most. Experiences of stigma may also contribute to avoidance strategies, such as avoiding social encounters and/or appointments, which in turn may further limit support and care. These are just some examples of the complex ways in which social identification may operate within long covid. Going forward, we recommend:

- Acknowledging that patients with long term conditions may experience challenging forms of "identity loss" which may lead them to feel more vulnerable.

- Addressing stigma in healthcare as well as in communities and workplaces, recognising that experiences of stigma are not always visible.

- Considering that stigma may have cumulative negative effects on recovery and may be more pronounced among already disadvantaged communities; that stigmatised groups may be less likely to seek support as a result of systemic bias.

- Approaching long covid from an integrated mind-body lens (as Ballering et al. suggest), believing the lived experience of patients and avoiding the discarding of somatic symptoms as psychological. $^{12}$

- Encouraging existing and new support groups to focus on effective ways of fostering positive 
identities, with a focus on the most vulnerable communities.

- Conducting interdisciplinary research where patients, social psychologists, epidemiologists, sociologists, clinicians, healthcare and public health practitioners, and those from the medical humanities, work together to understand long covid holistically, as insights can inform interventions that enhance positive social identities.

Competing interests: All authors have lived experience of long covid.

1 Prevalence of ongoing symptoms following coronavirus (COVID-19) infection in the UK. Office for National Statistics. https://www.ons.gov.uk/peoplepopulationandcommunity/healthandsocialcare/conditionsanddiseases/datasets/alldatarelatingtoprevalenceofongoingsymptomsfollowingcoronaviruscovid19infectionintheuk

2 Jetten J, Haslam SA, Cruwys T, Greenaway KH, Haslam C, Steffens NK. Advancing the social identity approach to health and well-being: Progressing the social cure research agenda. Eur J Soc Psychol 2017;47:789-802. doi: 10.1002/ejsp.2333.

3 Marija Pantelic and Nisreen Alwan. The stigma is real for people living with long covid. BMJ Opinion. https://blogs.bmj.com/bmj/2021/03/25/marija-pantelic-and-nisreen-alwan-the-stigmais-real-for-people-living-with-long-covid/

4 Abrams D, Houston DM, Van de Vyver J, Vasiljevic M. Equality hypocrisy, inconsistency, and prejudice: The unequal application of the universal human right to equality. Peace Confl 2015;21:28-46. doi: 10.1037/pac0000084. pmid: 25914516

5 FitzGerald C, Hurst S. Implicit bias in healthcare professionals: a systematic review. BMC Med Ethics 2017;18:19. doi: 10.1186/s12910-017-0179-8. pmid: 28249596

6 Baumeister RF, Leary MR. The need to belong: desire for interpersonal attachments as a fundamental human motivation. Psychol Bull 1995;117:497-529. doi: 10.1037/0033-2909.117.3.497 pmid: 7777651

7 Greenaway KH, Haslam SA, Cruwys T, Branscombe NR, Ysseldyk R, Heldreth C. From "we" to "me": Group identification enhances perceived personal control with consequences for health and well-being. J Pers Soc Psychol2015;109:53-74. doi: 10.1037/pspi0000019. pmid: 25938701

8 Cruwys T, South El, Greenaway KH, Haslam SA. Social Identity Reduces Depression by Fostering Positive Attributions. Soc Psychol Personal Sci2015;6:65-74. doi: 10.1177/1948550614543309.

9 van Dick R, Wagner U. Social identification among school teachers: Dimensions, foci, and correlates. Eur J Work Organ Psychol 2002;11:129-49. doi: 10.1080/13594320143000889.

10 Junker NM, van Dick R, Avanzi L, Häusser JA, Mojzisch A. Exploring the mechanisms underlying the social identity-ill-health link: Longitudinal and experimental evidence. Br J Soc Psychol 2019;58:991-1007. doi: 10.1111/bjso.12308. pmid: 30561049

11 Haslam C, Cruwys T, Haslam SA, Dingle G, Chang MX. Groups 4 Health: Evidence that a social-identity intervention that builds and strengthens social group membership improves mental health. J Affect Disord 2016;194:188-95. doi: 10.1016/j.jad.2016.01.010. pmid: 26828756

12 Ballering A, Olde Hartman T. Rosmalen LLong COVID-19, persistent somatic symptoms and social stigmatisation). Epidemiol Community Health 2021;75:603-4doi: 10.1136/jech-2021-216643. 